\title{
Detecting Vigabatrin Toxicity by Imaging of the Retinal Nerve Fiber Layer
}

\author{
Jobn M. Wild, ${ }^{1}$ Catberine R. Robson, ${ }^{1}$ Adrian L. Jones, ${ }^{2}$ Ian A. Cunliffe, ${ }^{3}$ and \\ Philip E. M. Smith ${ }^{4}$
}

Purpose. To quantify retinal nerve fiber layer thickness (RNFLT) and macular thickness (MT) in patients exhibiting vigabatrin-attributed visual field loss (VAVFL) and to determine the efficacy of these measures as markers of the retinal damage associated with vigabatrin.

Methods. This was a prospective cross-sectional observational study involving five groups: Group I, 13 patients exhibiting VAVFL; Group II, 8 patients exposed to vigabatrin but with normal fields; Group III, 14 patients receiving carbamazepine monotherapy; Group IV, 20 normal individuals; and Group V, 7 patients receiving sodium valproate monotherapy. At one of two visits, the right eye of each participant underwent two digital imaging modalities: ocular coherence tomography (OCT; StratusOCT; Carl Zeiss Meditec, Dublin, CA) and scanning laser ophthalmoscopy (SLO; Heidelberg Retinal Tomograph; Heidelberg Engineering $\mathrm{GmbH}$, Heidelberg, Germany). At the other visit, participants underwent three-zone, agecorrected suprathreshold perimetry of the whole field and threshold perimetry of the central field (Humphrey Field Analyzer 750; Carl Zeiss Meditec). The order of the visits was randomized.

Results. The group mean RNFLT in Group I was attenuated relative to that of the remaining groups (all $P<0.001$ ). At $100 \%$ specificity, based on the $95 \%$ confidence limits derived from Group IV, OCT exhibited 100\% sensitivity and SLO $77 \%$ sensitivity for an attenuated RNFLT in patients with VAVFL. All participants manifested an MT within the normal range derived from Group IV.

Conclusions. OCT of the RNFL can efficiently identify vigabatrin-induced damage and will be useful for adults and children unable to perform perimetry and when the perimetric outcome is equivocal. (Invest Ophthalmol Vis Sci. 2006;47: 917-924) DOI:10.1167/iovs.05-0854

$\mathrm{V}$ igabatrin, the first of the novel anti-convulsants, is used in approximately 85 countries outside the United States, as adjunctive therapy for the treatment of epilepsy of partial onset $^{1-3}$ and as monotherapy for infantile spasms, ${ }^{4,5}$ particu-

From the ${ }^{1}$ Cardiff School of Optometry and Vision Sciences, Cardiff University, Cardiff, Wales, United Kingdom; the ${ }^{2}$ Cardiff Eye Unit and the ${ }^{4}$ Welsh Epilepsy Unit, University Hospital of Wales, Cardiff, Wales, United Kingdom; and the ${ }^{3}$ Department of Ophthalmology, Birmingham Heartlands Hospital, Birmingham, United Kingdom.

Presented in part at the 57th Annual Meeting of the American Academy of Neurology, Miami Beach, Florida, April 9-16, 2005.

Submitted for publication July 2, 2005; revised August 25, 2005; accepted January 4, 2006.

Disclosure: J.M. Wild, Carl Zeiss Meditec (R), Sanofi-Aventis (R, C); C.R. Robson, None; A.L. Jones, None; I.A. Cunliffe, None; P.E.M. Smith, None

The publication costs of this article were defrayed in part by page charge payment. This article must therefore be marked "advertisement" in accordance with 18 U.S.C. $\$ 1734$ solely to indicate this fact

Corresponding author: John M. Wild, Cardiff School of Optometry and Vision Sciences, Cardiff University, King Edward VII Avenue, Cathays Park, Cardiff CF 10 3NB, Wales, UK; wildjm@cf.ac.uk. larly those secondary to tuberous sclerosis. ${ }^{6}$ Vigabatrin is a selective and irreversible inhibitor of the enzyme $\gamma$-aminobutyric acid (GABA)-transaminase, which catalyzes the inactivation of GABA, a major inhibitory neurotransmitter in the retina and cortex. ${ }^{7}$ The resultant anticonvulsant effect of vigabatrin is thought to occur from the increase in concentration of presynaptic GABA.

Vigabatrin is associated with a bilateral constriction of the visual field. $^{8-15}$ The estimated prevalence of vigabatrin-attributed visual field loss ranges from $14 \%{ }^{16}$ to $92 \%{ }^{17}$ but is generally considered to be in the region of $40 \%$ to $50 \% .^{12,13,18,19}$ Patients with vigabatrin-attributed field loss exhibit normal visual acuity and are usually asymptomatic of the field loss unless the defect encroaches well within the central field. ${ }^{8,10-15}$ Approximately 20\% of adults with epilepsy, and particularly those exposed to vigabatrin, are unable to appreciate the requirements of perimetry due to the cognitive requirements for, and the subjective nature of, the task. ${ }^{20}$ In many others, the results of perimetry can often be inconclusive and frequently require one or more confirmatory repeat examinations, even though the results of the subsequent tests can remain equivocal.

Vigabatrin-attributed field loss can exist in the presence of an apparently normal optic nerve head and retina ${ }^{12,21}$ or can be associated with optic nerve head pallor ${ }^{11,15,22,23}$ with or without a variety of accompanying subtle retinal abnormalities including surface wrinkling retinopathy ${ }^{9,23}$ peripheral retinal arterial narrowing ${ }^{9,15}$; irregular sheen, or abnormal pigmentation, at the macula9; peripheral pigmentary disturbance ${ }^{14,15}$; and thinning of either the peripapillary ${ }^{13}$ or peripheral ${ }^{22,23}$ retinal nerve fiber layer. The inconclusive and subtle nature of any coexisting optic nerve head and retinal abnormalities precludes the use of fundal examination by ophthalmoscopy as an indicator of vigabatrin-attributed field loss.

The postmortem examination of the retina of a patient with vigabatrin-attributed field loss has indicated loss of rod and cone nuclei and extensive loss of retinal ganglion cells and their axons. ${ }^{24}$ An attenuated retinal nerve fiber layer has been described in one patient with vigabatrin-attributed visual field loss, detected by optical coherence tomography (OCT), ${ }^{25}$ and in another by confocal scanning laser ophthalmoscopy (SLO). ${ }^{26}$ However, although likely to be of particular use in the assessment of vigabatrin-induced damage, digital imaging technology of the ocular fundus has yet to be applied in a systematic manner to patients receiving antiepileptic drugs.

The overall aim of the present study, therefore, was to investigate the potential of retinal imaging to indicate vigabatrin-attributed field loss. The specific aims were to quantify, in patients with vigabatrin-attributed field loss and in patients exposed to vigabatrin but with normal fields, retinal nerve fiber layer and macular thicknesses by OCT, and the retinal nerve fiber layer thickness by SLO, thereby determining whether any of these measures could be used as a marker for vigabatrinattributed retinal damage. The two different imaging modalities were evaluated to determine which technique, if any, possessed the better sensitivity and specificity. If structural mark- 
ers could be determined, it is possible that such changes might occur before the visual field loss became established.

\section{Methods}

The study was of a cross-sectional prospective observational design.

\section{Cohort}

The cohort comprised five groups of consecutively presenting patients and normal individuals, aged 18 years or older, who had volunteered to take part in the study after invitation by letter. Group I comprised 13 patients with epilepsy of various etiologies who had been or were currently, exposed to vigabatrin and manifested vigabatrin-attributed field loss. Group II comprised eight patients with epilepsy who had been or were currently, exposed to vigabatrin and manifested normal visual fields. Two additional patients exposed to vigabatrin exhibited equivocal visual fields and therefore could not be categorized in either Group I or II. Group III comprised 14 patients with epilepsy who had never been exposed to vigabatrin and who, at the time of the study, were receiving carbamazapine monotherapy. Carbamazepine is an anti-epileptic drug; the primary mechanism of action is believed to be the blockade of voltage-dependent sodium channels. The patients in Group III therefore served as the control for the effects of GABA modulation. Group IV comprised 20 clinically normal individuals who did not have epilepsy and who had not been exposed to antiepileptic drugs. These individuals served as a basis for the creation of appropriate confidence limits for normality for the results from the retinal imaging. Group V comprised seven patients with epilepsy who were included in the study after the data for the remaining four groups had been analyzed, and it had become apparent that patients in Groups I and II had frequently been treated with the antiepileptic drug sodium valproate before, or as combination therapy with, vigabatrin. Valproate has a mild GABAergic action believed to result from inhibition of GABA-transaminase and of succinic semialdehyde dehydrogenase and from the stimulation of benzymeglutamic acid dehydrogenase which synthesizes GABA. The patients in Group V had been treated with valproate monotherapy and served, as a post hoc control, for those patients in Group I who had been treated with valproate.

All patients were recruited from the Welsh Epilepsy Unit, University Hospital of Wales, Cardiff, and the normal individuals from the University Hospital of Wales and from the Eye Clinic, Cardiff School of Optometry and Vision Sciences, Cardiff University. The participants were matched as closely as possible in age within and between the respective groups. As far as possible, patients were matched for age at onset, and duration, of epilepsy.

All participants had undergone ocular examination and conformed in each eye to rigid inclusion criteria, including a distance refractive error less than or equal to 5 diopters mean sphere and less than 2.5 diopters cylinder; open angles, and clear ocular media; no optic nerve head or fundal abnormalities characteristic of known disease; no previous ocular surgery or trauma; no history of diabetes mellitus and no family history of glaucoma. All participants manifested a visual acuity of $20 / 30$ or better in each eye and an intraocular pressure of $21 \mathrm{~mm} \mathrm{Hg}$ or less.

The participants attended two further visits. At one visit, they underwent visual field examination of the right eye. At the other, they underwent retinal imaging in the same eye. The order of the imaging and perimetry visits was randomized between individuals.

\section{Perimetry}

The visual field examination was performed with the Humphrey Visual Field Analyzer 750 (Carl Zeiss Meditec, Dublin, CA). Three-zone, agecorrected suprathreshold perimetry was undertaken with the Full Field 135 Point Screening Program, followed by threshold perimetry undertaken with Program 30-2 and the FASTPAC strategy. The appropriate refraction, corrected for the viewing distance of the perimeter bowl, was used during the examination of the central field. No correction was used for the examination beyond $30^{\circ}$ from fixation. Patients were given frequent rest periods throughout each perimetric examination and a break of 15 to 30 minutes between examinations.

If either of the visual field examinations for any given patient were inconclusive, the corresponding examination was repeated at a subsequent visit. One patient with an unequivocal normal field derived by the Full Field 135 Point Screening Program was unable to complete Program 30-2.

Each participant in the five groups exhibited stable fixation for each perimetric examination, as indicated by the response to the gaze tracker and the responses to the fixation loss catch trials. The incorrect responses to the false-positive catch trials were also within the normal range $(<30 \%)$ for all participants. The incorrect responses to the false-negative catch trials were within the normal range $(<30 \%)$ for all individuals in Groups II through V. In Group I, two patients exhibited incorrect responses beyond the normal range for the Full Field 135 Point Screening Program, one participant for Program 30-2, and one for both programs.

\section{Imaging}

The imaging visit consisted of retinal imaging with OCT (StratusOCT, Carl Zeiss Meditec) and SLO with the Heidelberg Retina Tomograph II (HRT; Heidelberg Engineering, Dossenheim, Germany).

For OCT, each participant first underwent the Fast Optic Disc scan, centered on the optic disc, from which the vertical diameter of the optic nerve head was obtained. The participants then underwent three separate $360^{\circ}$ circular scans, centered on the optic disc, using the Proportional Circle scan incorporating a scan radius that corresponded to the vertical diameter of the optic nerve head, thereby accounting for between-subject differences in the size of the optic nerve head. The macula was then separately imaged using the Macular Thickness Map scan and the Fast Macular Thickness Map scan. The two types of Macular Thickness Map scan were undertaken to determine whether the Fast Macular Thickness Map scan exhibited equivalent sensitivity and specificity relative to the longer acquisition time and increased resolution of the Macular Thickness Map scan.

The contralateral eye was occluded and participants fixated on the internal fixation target. The $z$-offset and polarization were optimized before each Proportionate Circle and Macular Thickness Map scan was acquired. All scans exhibited the requirements of a signal-to-noise ratio greater than $25 \mathrm{~dB}$ and at least $90 \%$ good-quality A-scans. The images were then each analyzed by StratusOCT software version 3.0.

For SLO, the corneal radius was determined before imaging with a keratometer (Bausch \& Lomb, Rochester, NY) to correct the images for ocular magnification. Three separate scans of the optic nerve head, and the immediate surrounding retina, were automatically obtained by the HRT software which then computed the mean of the three scans to form the output topographic image and the SD of the mean to ascertain the quality of the resulting mean image. The field size was $15^{\circ} \times 15^{\circ}$. The participants fixated on the internal fixation target. The SD of the mean was $\leq 10 \mu \mathrm{m}$ in 9 of the 55 participants, between 11 and $20 \mu \mathrm{m}$ in 36 , between 21 and $30 \mu \mathrm{m}$ in 9 participants, and $57 \mu \mathrm{m}$ in the remaining participant in Group III. The contour line was drawn by a senior ophthalmologist (IAC) trained to fellowship standard in glaucoma and highly experienced in optic nerve head assessment and in the drawing of the contour line with the HRT, who was masked to the purpose and design of the study. The images were then analyzed by HRT software version 1.6.

The order of the imaging modality was randomized between the participants. The right eye of each participant was dilated with $0.5 \%$ tropicamide before imaging, to ensure a minimum pupil diameter of $5 \mathrm{~mm}$.

\section{Analysis}

The primary analysis was undertaken on the results from the patients in Groups I through IV. After completion of the data collection for the groups, the visual field results from each participant were evaluated by 
TABLE 1. The Summary Biographical Data for Each of the Five Groups

\begin{tabular}{lccccc}
\hline Group & $\begin{array}{c}\text { Proportion Male: } \\
\text { Female }\end{array}$ & $\begin{array}{c}\text { Age (SD) } \\
\text { (y) }\end{array}$ & $\begin{array}{c}\text { Duration of } \\
\text { Epilepsy (SD) } \\
\text { (ys) }\end{array}$ & $\begin{array}{c}\text { Duration of Vigabatrin } \\
\text { Therapy (SD) } \\
\text { (kg) }\end{array}$ & $\begin{array}{c}\text { Cumulative Dose } \\
\text { of Vigabatrin (SD) } \\
\text { (kg) }\end{array}$ \\
\hline I & $7: 6$ & $42.9(8.9)$ & $27.3(10.8)$ & $10.1(1.9)$ & $7.8(2.7)$ \\
II & $8: 0$ & $40.1(10.8)$ & $25.9(16.8)$ & $7.3(4.2)$ & Nil \\
III & $3: 11$ & $41.2(8.8)$ & $19.5(15.3)$ & Nil & Nil \\
IV & $5: 15$ & $39.7(12.3)$ & Nil & Nil & Nil \\
V & $4: 3$ & $35.0(11.2)$ & $21.3(14.8)$ & & Nil \\
\hline
\end{tabular}

Data are the group mean $\pm \mathrm{SD}$.

one of the authors (JMW), who was aware of the categorization of participants into one of four potential groups but was masked to all the remaining clinical information including the therapeutic history and the results of the retinal imaging. No patients in Group III and no individuals in Group IV manifested visual field loss. The post hoc inclusion of the patients in Group V meant that the evaluator was aware that these patients were receiving valproate; however, he was masked to the results of the retinal imaging. All seven patients exhibited normal fields.

For OCT, the retinal nerve fiber layer thickness of individual participants in each group was compared with the lower 95th confidence limit calculated from the individuals in Group IV and derived from the group mean $\pm t \times \mathrm{SD}$ where $t$ is the appropriate percentile of the $t$ distribution with $n-1$ degrees of freedom. Separate analysis of covariance (ANCOVA) was undertaken for Groups I to IV on the average thickness for the complete $360^{\circ}$ scan and for the four sectors, superior, inferior, nasal, and temporal. The mean of the three separate Proportional Circle scans for each participant was used for each analysis. For each of the two data sets, group was considered as a betweensubjects factor and age as the covariate. Suitable linear contrasts were then used to compare the average thickness between pairs of groups, corrected for any age differences. Inferential analysis was not undertaken for the thickness in $30^{\circ}$ segments due to the likelihood of Type I errors arising from vast number of multiple comparisons.

Results for individual participants for both types of OCT Macular Thickness Map scan were compared with the corresponding normal limits calculated from the individuals in Group IV. Separate ANCOVAs were undertaken for both types of scan, using the weighted average thickness according to the standard weighting function. ${ }^{27}$ Linear contrasts were also used to compare the weighted average thickness between pairs of groups, corrected for any age differences.

For HRT, the results for the individuals in each group were compared with the lower 95th confidence limits calculated from the normal individuals in Group IV. Separate ANCOVAs were undertaken for the mean nerve fiber layer thickness at the radial extremity along the entire contour line and for the mean nerve fiber layer thickness for each of six sectors: nasal, temporal, superior nasal, superior temporal, inferior nasal, and inferior temporal. Linear contrasts compared the mean overall thickness, and also the mean thickness for each of six sectors, between pairs of groups, corrected for any age differences.

The research complied with the tenets of the Declaration of Helsinki. All individuals were provided with verbal and written information concerning the nature of the study and gave written informed consent after explanation of the nature and possible consequences of the study, in accordance with the guidelines of the Local Research and Ethics Committee.

\section{Results}

The respective biographical data (group mean and SD) for each of the five groups are listed in Table 1 . At the time of the study, six patients in Group I and one patient in Group II were being treated with vigabatrin. The mean duration of vigabatrin therapy was greater in Group I than in Group II $(P=0.049)$, but the mean cumulative dose of vigabatrin was similar between the two groups $(P=0.337)$.

In the patients in Group III, the mean period of monotherapy with carbamazepine was $1.0 \pm 1.2$ years (SD) with a minimum period of 6 months. Five of the 14 patients had been on monotherapy throughout their clinical care. Two patients had each been treated with sodium valproate for 4 years and one patient with the antiepileptic drug phenytoin for 6 years. The remainder had received a variety of non-GABAergic antiepileptic drugs that had been withdrawn due to intolerance or ineffectiveness after short periods ranging from 12 days to 5 months. The mean duration of carbamazepine therapy was $6.1 \pm 6.4$ years, and the mean cumulative dose was $1.19 \pm 1.3$ $\mathrm{kg}$ (SD).

The age of the patients was similar between the four groups $(P=0.847)$. The apparent difference in the duration of epilepsy between the patients in Groups I and II and those in Group III did not reach statistical significance $(P=0.430)$.

Six of the seven patients in Group $V$ had received valproate monotherapy throughout their clinical care. The seventh patient had received the antiepileptic drug lamotrigine as add-on therapy for approximately 5 months, 6 years previously. The mean duration of valproate therapy was $7.9 \pm 3.6$ years, and the mean cumulative dose was $3.44 \pm 2.2 \mathrm{~kg}$.

\section{Optical Coherence Tomography}

The average retinal nerve fiber layer thickness for the complete $360^{\circ}$ scan for each participant as a function of group is given in Figure 1. All patients with vigabatrin-attributed visual field loss exhibited a nerve fiber layer thickness beyond the lower 95\% confidence limit for normality (i.e., 100\% sensitivity at 100\% specificity) Three of the eight patients exposed to vigabatrin and manifesting normal visual fields exhibited an apparently abnormal nerve fiber layer thickness.

Average retinal nerve fiber layer thickness for the complete $360^{\circ}$ scan varied across Groups I through IV $(P<0.001$; Table 2). The mean average thickness in Group I, $64.8 \mu \mathrm{m}$, was attenuated relative to that of Group II, 97.1 $\mu \mathrm{m}(P<0.001)$; Group III, $101.5 \mu \mathrm{m}(P<0.001)$; and Group IV, $110.6 \mu \mathrm{m}(P<$ 0.001). Given the limited size of the study sample and given the tendency for an increased likelihood of a Type I statistical error resulting from the multiple comparisons of means, there was some evidence to suggest that the mean average thickness in Group II was attenuated relative to that of Group IV $(P<$ 0.022). However, this latter outcome was influenced by the two patients in Group II with an average thickness lying outside the $95 \%$ confidence limits. The trend in the average thickness between Groups I and II was repeated for each of the superior, inferior, and nasal sectors (Table 2).

The average nerve fiber layer thickness for the complete $360^{\circ}$ scan in patients exposed to vigabatrin as a function of the Mean Sensitivity derived with Program 30-2 is shown in Figure 2 and as a function of duration of therapy with vigabatrin and of a cumulative dose of vigabatrin in Figure 3. 


\section{Retinal nerve fiber layer thickness derived by OCT}

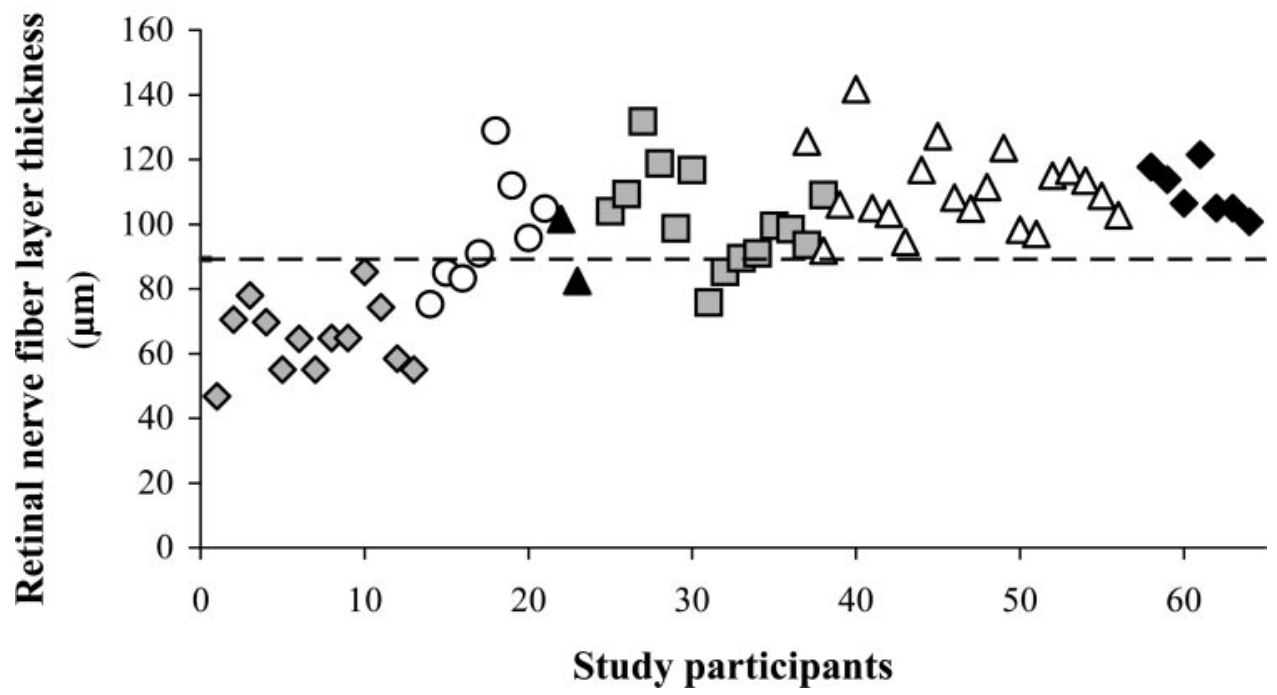

Figure 1. The average retinal nerve fiber layer thickness (in micrometers) derived by OCT, for the complete $360^{\circ}$ scan in each of the participants in each of the five groups. Shaded diamonds: patients exhibiting vigabatrin-attributed visual field loss; open circles: patients exposed to vigabatrin but with normal visual fields; shaded squares: patients receiving carbamazapine monotherapy; open triangles: normal individuals; and filled diamonds: patients receiving sodium valproate monotherapy. The lower 95 th percentile for normality is illustrated by the dasbed line. Two patients exposed to vigabatrin (indicated by the filled triangles) each exhibited equivocal visual fields.

All patients, regardless of group, exhibited a weighted mean macular thickness within the $95 \%$ confidence limits of normality for either type of scan derived from the participants in Group IV. The group means for the weighted mean macular thickness in the Fast Macular Thickness Map scan and in the Macular Thickness Scan did not differ across Groups I through IV for either type of scan $(P=0.086)$ and $(P=0.086)$, respectively.

Of the two patients exposed to vigabatrin who exhibited equivocal visual fields, one manifested an abnormal nerve fiber layer thickness and a normal macular thickness. The other manifested normal nerve fiber layer and macular thicknesses.

\section{Scanning Laser Ophthalmoscopy}

Ten of the 13 patients with vigabatrin-attributed visual field loss exhibited a mean nerve fiber layer thickness derived by SLO beyond the $95 \%$ confidence limits for normality (i.e., $76.9 \%$ sensitivity at $100 \%$ specificity; Fig. 4). Two of the eight patients exposed to vigabatrin and manifesting normal visual fields exhibited an apparently abnormal nerve fiber layer thickness.

The mean nerve fiber layer thickness derived by SLO (Table $3)$ varied across Groups I to IV $(P<0.001)$. The mean Mean
Thickness in Group I, $0.126 \pm 0.05$ (SD) was attenuated relative to that of Group II, $0.210 \pm 0.10 \mathrm{~mm}(P<0.034)$; Group III, $0.290 \pm 0.10(P<0.001)$; and Group IV, $0.260 \pm$ $0.06 \mathrm{~mm}(P=0.001)$. The trend in the overall thickness between Groups I and II for each of the six sectors was less convincing and not as profound as the sector analysis for OCT (Table 2).

Of the two patients exposed to vigabatrin who exhibited equivocal visual fields, one manifested an abnormal and the other a normal nerve fiber layer thickness.

\section{Discussion}

The results provide evidence of an attenuated retinal nerve fiber layer thickness in patients with vigabatrin-attributed visual field loss. Moreover, the sensitivity and specificity of the digital imaging techniques used in the study, particularly that of OCT, combined with the objective nature and relatively short chair time, suggest that the technique may be considered in clinical practice for the assessment of vigabatrin-attributed damage. In addition, the technique is also advocated for the evaluation of potential structural damage to the retina by ex-

Table 2. The Probabilities for the Comparison of the Mean Average RNFL Thickness with the Mean Sector Average RNFL Thickness for the Complete $360^{\circ}$ Scan

\begin{tabular}{lccccc}
\hline Group Comparison & $360^{\circ}$ & Superior & Inferior & Nasal & Temporal \\
\hline I v II & $P<0.001$ & $P<0.001$ & $P<0.001$ & $P<0.001$ & $P=0.016$ \\
I v III & $P<0.001$ & $P<0.001$ & $P<0.001$ & $P<0.001$ & NS \\
I v IV & $P<0.001$ & $P<0.001$ & $P<0.001$ & $P<0.001$ & $P<0.001$ \\
II v III & NS & NS & NS & NS & NS \\
II v IV & $P=0.022$ & $P=0.039$ & $P=0.044$ & $P=0.025$ & NS \\
III v IV & NS & NS & NS & NS & NS
\end{tabular}

Data were derived by OCT in each of Groups I through IV. 


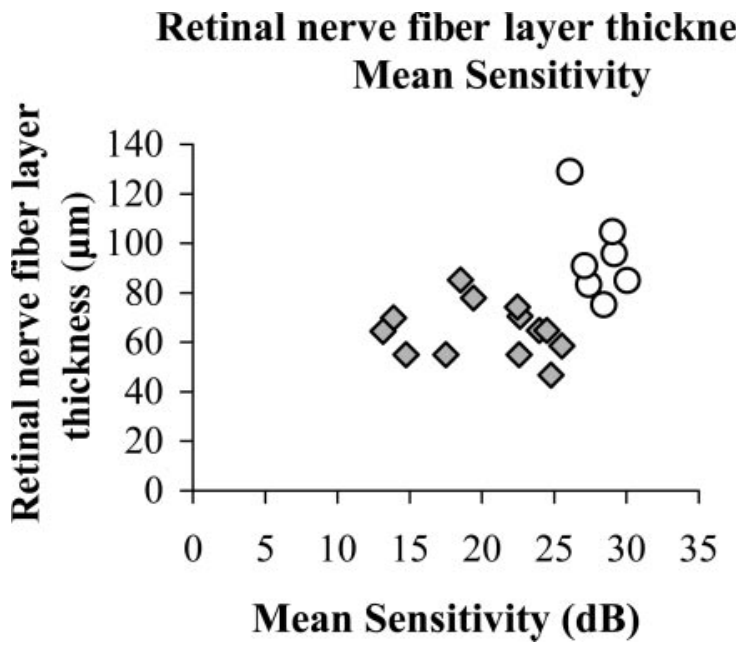

FIGURE 2. The average retinal nerve fiber layer thickness (in micrometers) for the complete $360^{\circ}$ scan derived by OCT against Mean Sensitivity (in decibels) derived with Program 30-2 in the patients with vigabatrin-attributed visual field loss (Group I; diamonds) and for the patients exposed to vigabatrin but with normal fields (Group II; circles). Note that one patient with unequivocally normal fields did not complete Program 30-2 and therefore cannot be included in the figure.

isting GABAergic antiepileptic drugs and for those in Phase III studies.

The visual electrophysiology associated with vigabatrin is complex but suggests a retinal rather than a cortical origin for vigabatrin-attributed field loss. Vigabatrin is associated with a reduced Arden Index of the electrooculogram (EOG) ${ }^{10,28,29}$ and abnormalities of the electroretinogram (ERG) including reduced cone b-wave, ${ }^{9,30}$ a decreased amplitude of the $30-\mathrm{Hz}$ flicker response, ${ }^{20,23}$ and abnormalities in photopic and scotopic oscillatory potentials..$^{9,11,13,30,31}$ Separation of the electrophysiological effects due to vigabatrin therapy from those associated with vigabatrin-attributed damage, implicates an abnormal cone function in association with the field loss. ${ }^{20}$ However, although the latencies of the photopic a-wave and of the $30 \mathrm{~Hz}$ flicker a-wave and the $30 \mathrm{~Hz}$ flicker a-b amplitude yield encouraging sensitivities for the detection of at least severe vigabatrin-attributed field loss, the accompanying specificities are not sufficiently high to justify implementation of the technique. ${ }^{20}$ Wide-field multifocal electroretinography may yield better outcomes in this regard. ${ }^{32}$ The presence of functional abnormality at the fovea, such as reduced contrast sensitivity ${ }^{33,34}$ and abnormal color vision ${ }^{13,32,34,35}$ in patients exposed to vigabatrin is also equivocal and, therefore, measurement of these functions cannot be used to indicate vigabatrinattributed field loss.

All patients in Groups I and II had received a variety of antiepileptic drugs encompassing the complete range of available therapies. However, eight of the 13 patients with vigabatrin-attributed visual field loss had been treated with sodium valproate before therapy with vigabatrin and four of these eight had received combination therapy of valproate and vigabatrin. The mean duration of therapy with valproate in this group was $10.0 \pm 6.2$ years. Six of the eight patients had been treated with valproate for nine years or more. In contrast, only two of the eight patients in Group II had received valproate: one for almost 5 years before therapy with vigabatrin and the other for 16 years of which 11 years included combination therapy with vigabatrin. One patient in Group II received valproate after withdrawal of vigabatrin. It is not possible to attribute, unequivocally, the attenuation of the nerve fiber layer to vigabatrin. Nevertheless, the evidence for a purely vigabatrin etiology is persuasive. The evidence from the study is three fold. Firstly, all patients in Group I exhibited field loss unequivocally characteristic of vigabatrin irrespective of exposure to valproate. Second, the two patients with long-term exposure to valproate in Group II both exhibited normal fields. Finally, all seven patients in Group V receiving valproate monotherapy exhibited a nerve fiber layer thickness well within the normal range by both OCT (group mean $110.0 \pm 7.7 \mu \mathrm{m}$ [SD]) and SLO (group mean $0.273 \pm 0.06 \mathrm{~mm}[\mathrm{SD}]$ ). The evidence from the literature is also convincing. Characteristic vigabatrinattributed visual field loss has been observed with vigabatrin monotherapy ${ }^{12}$ and, in the most authoritative study of valproate monotherapy and visual function, involving 32 patients treated for a mean duration of 6 years and using a daily dose between 1000 and $2000 \mathrm{mg}$, visual acuity, color vision, central field, and scotopic and photopic ERGs were all normal. ${ }^{36}$ The drug history of the patients in Groups I and II is believed to reflect the local idiosyncrasy of the prescribing practice in the Welsh Epilepsy Unit during the early to mid 1990s when valproate was prescribed as the drug of first choice for all types

\section{Retinal nerve fiber layer thickness and Exposure Duration}
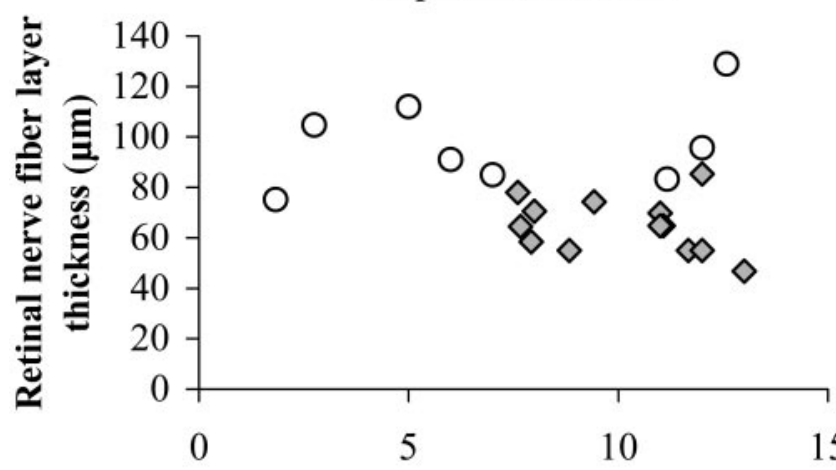

Duration of treatment (Yrs)

\section{Retinal nerve fiber layer thickness and Cumulative Dose}

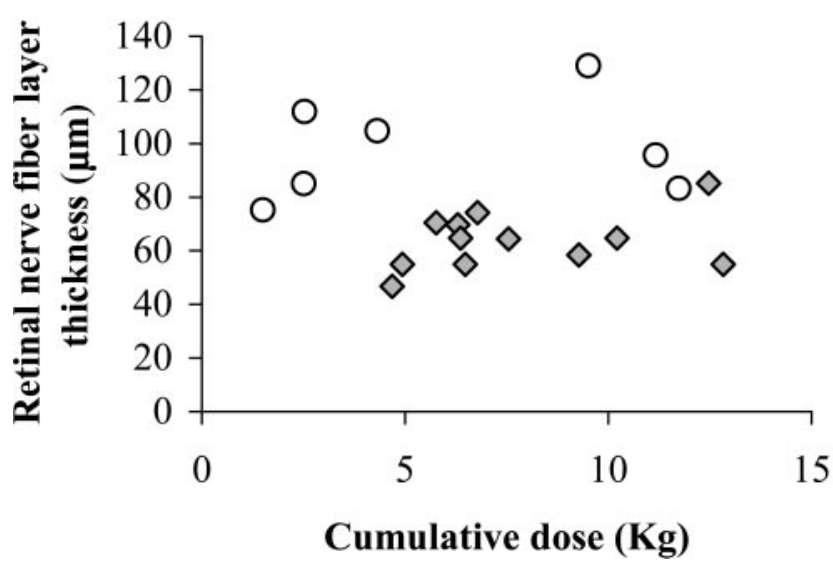

FIGURE 3. The average retinal nerve fiber layer thickness (in micrometers) for the complete $360^{\circ}$ scan derived by OCT as a function of (top) duration of therapy (years) with vigabatrin and (bottom) cumulative dose of vigabatrin (kilograms) for the patients with vigabatrin-attributed visual field loss (Group I; diamonds) and for the patients exposed to vigabatrin but with normal fields (Group II; circles). Note that the cumulative dose could not be determined accurately in one patient in Group II, and the value has been omitted from Figure 3 (bottom). 


\section{Retinal nerve fiber layer thickness derived by SLO}

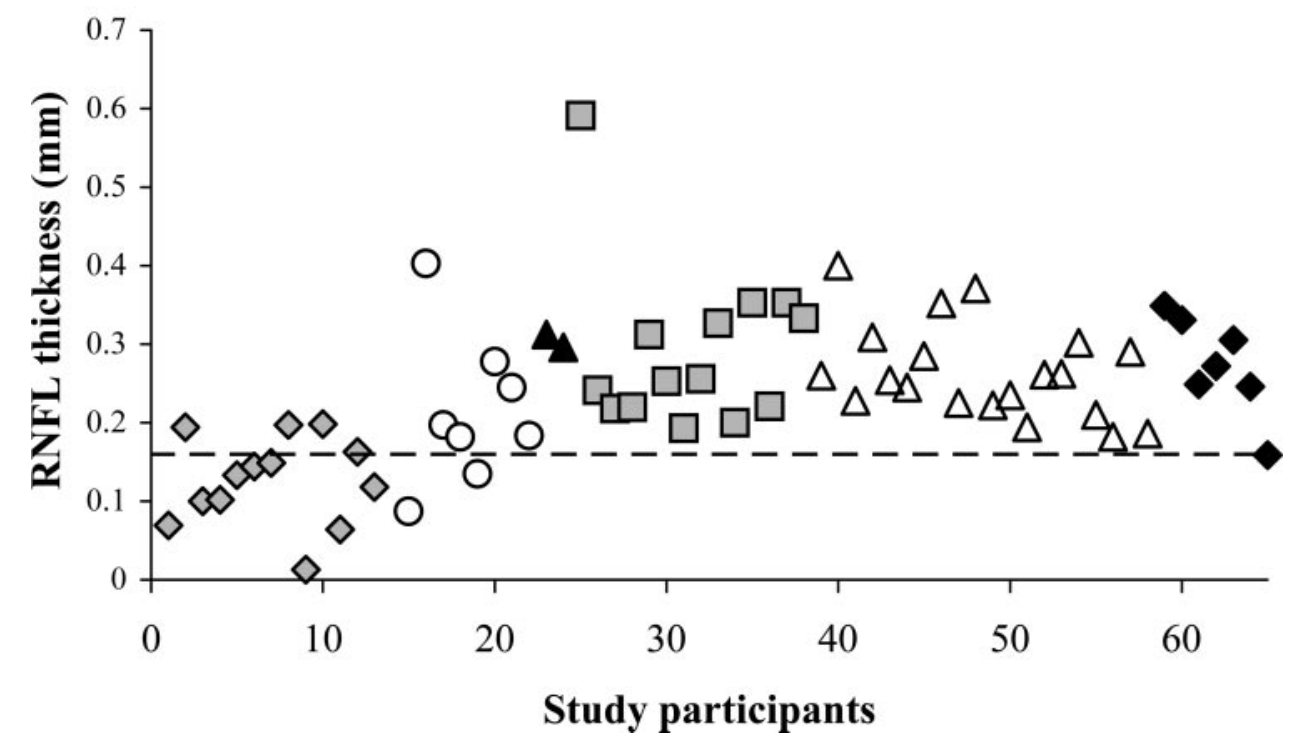

FIGURE 4. The mean retinal nerve fiber layer thickness (in millimeters) derived by SLO for each of the participants in each of the five groups. Shaded diamonds: patients exhibiting vigabatrin-attributed visual field loss; open circles: patients exposed to vigabatrin but with normal visual fields; shaded squares: patients receiving carbamazapine monotherapy; open triangles: normal individuals; and filled diamonds: patients receiving sodium valproate monotherapy. The lower 95 th percentile for normality is illustrated by the dashed line. Two patients exposed to vigabatrin (indicated by the filled triangles) exhibited equivocal visual fields.

of epilepsy and vigabatrin as the first choice add-on therapy for partial refractory epilepsy. Nevertheless, vigabatrin-attributed damage may be greater for combination therapy with valproate compared to combination therapy with carbamazepine. ${ }^{37}$ It may well be that such an effect is more profound with the long durations of valproate, and/or vigabatrin, encountered in the patients in Group I.

The time to occurrence of an attenuated retinal nerve fiber layer thickness resulting from vigabatrin therapy is unknown as is the extent of any further attenuation over time. Three patients exposed to vigabatrin but with normal fields manifested an abnormal average nerve fiber layer thickness for the complete $360^{\circ}$ scan by OCT. Similarly, two patients exposed to vigabatrin but with normal fields manifested an abnormal mean nerve fiber layer thickness by SLO. One of the patients exhibited abnormality by both techniques. These findings suggest that digital ocular imaging may be a more sensitive indicator than perimetry for vigabatrin-attributed damage. This is not surprising, given that retinal nerve fiber layer defects occur frequently in glaucoma, for example, before the emergence of a visual field defect. ${ }^{38}$

The two patients who received carbamazepine therapy and manifested an abnormal average retinal nerve fiber layer thick- ness by OCT did not exhibit any unusual clinical characteristics. Their retinal nerve fiber layer thickness was normal by SLO. The finding cannot be explained.

Despite the frequent characteristic binasal appearance of vigabatrin-attributed visual field loss within the central field out to $30^{\circ}$ eccentricity, the corresponding selectively greater attenuation in the temporal retinal nerve fiber layer thickness by OCT was not present in the four-sector analysis or in the graphic representation of the $30^{\circ}$ sector analysis. Similarly, no differences were found in the HRT results. Potential functional and structural correlation is likely to be confounded by several factors, including the lack of axial resolution of the segmental analysis contained on the respective OCT and HRT printouts, the lack of knowledge concerning the precise configuration between the given visual field stimulus location and the point of entry into the optic nerve head by the corresponding ganglion cell axons, ${ }^{39}$ and the between-individual variability in this correspondence. $^{39}$

The HRT results for the retinal nerve fiber layer thickness exhibited proportionately greater between-subject variability than the OCT in Groups III and IV. The greater variability is likely to stem from the reliance of the technique on the designation of the contour line by the clinician.

TABLE 3. The Probability Values for the Comparison of the Mean Overall RNFL Thickness with the Mean Sector RNFL Thickness Derived by SLO

\begin{tabular}{|c|c|c|c|c|c|c|c|}
\hline Group Comparison & $360^{\circ}$ & Superior Nasal & Superior Temporal & Nasal & Temporal & Inferior Nasal & Inferior Temporal \\
\hline I vs. II & $P=0.034$ & NS & NS & $P=0.031$ & $P=0.026$ & NS & $P=0.032$ \\
\hline I vs. III & $P<0.001$ & $P<0.001$ & $P<0.001$ & $P<0.001$ & NS & $P=0.003$ & $P=0.004$ \\
\hline I vs. IV & $P<0.001$ & $P<0.001$ & $P=0.001$ & $P<0.001$ & $P=0.046$ & $P=0.023$ & $P=0.021$ \\
\hline II vs. III & NS & $P=0.028$ & NS & $P=0.019$ & NS & NS & NS \\
\hline II vs. IV & NS & NS & NS & NS & NS & NS & NS \\
\hline III vs. IV & NS & NS & NS & NS & NS & NS & NS \\
\hline
\end{tabular}


The mean average nerve fiber layer thickness measured by OCT exhibits an apparent floor effect at approximately $47 \mu \mathrm{m}$. However, it should be noted that the papillomacular bundle of ganglion cell fibers is seemingly unaffected by vigabatrin ${ }^{23}$ and thus will contribute to the average value for the nerve fiber layer thickness. The minimum individual recorded thickness for the thinnest sector, the nasal sector was $30 \mu \mathrm{m}$.

The retinal nerve fiber layer thickness decreases with increasing eccentricity from the optic nerve head. ${ }^{40-42}$ Two concepts exist for the selection of a circular scan for OCT. The first advocates a fixed diameter circular scan of $3.46 \mathrm{~mm}$. This diameter is large enough to prevent any overlap with the optic nerve head in nearly all eyes and combines measurement of a reasonably thick area of the RNFL with optimum reproducibility. ${ }^{40}$ The normative database contained within the StratusOCT is based on such a diameter. However, the use of a fixed diameter scan is highly dependent on the axial length and, to a lesser extent, on the refractive power of the eye being imaged and does not account for variation in optic disc size within the population. A fixed-diameter scan therefore measures the nerve fiber layer thickness closer to the optic disc border in larger discs than in smaller ones. ${ }^{42}$ The second approach, adopted in the present study, utilizes a scan radius based on an increment of the vertical disc diameter; in this instance, unity. Such an approach overcomes the variation in the size of the optic nerve head. The choice of the increment is arbitrary. A scan radius based on an increment of unity yielded a mean normal average nerve fiber layer thickness of $110.6 \mu \mathrm{m}$. A scan radius closer to the optic nerve head (i.e., an increment of less than unity) would have yielded a thicker measure of the retinal nerve fiber layer which would have enabled a greater measurement range. The use of a scan radius based on an increment of the vertical disc diameter would be appropriate for the follow-up of children and adolescents.

The normal macular thickness in all patients in Groups I and II indicates that, within the resolution of the OCT, vigabatrin was not associated with a disturbance of the macula. There was no evidence of epiretinal membrane formation within the patients in Groups I or II. SLO was not used to evaluate the macula because, at the time of the study, the software permitting evaluation of the macula was not commercially available.

In summary, digital imaging of the retina by two different optical techniques, OCT and SLO, yielded an attenuated retinal nerve fiber layer thickness for patients with vigabatrin-attributed visual field loss. Macular thickness was normal by OCT. Assessment of retinal nerve fiber layer thickness, particularly by OCT, is a clinically viable indicator of vigabatrin-attributed damage and may be considered as an adjunct to the assessment of all adult patients exposed to vigabatrin, particularly the learning disabled and those in whom the visual field result is equivocal. Wherever possible, it should also be considered for children exposed to vigabatrin.

\section{References}

1. Ben-Menachem E, Persson LI, Schechter PJ, et al. The effect of different vigabatrin treatment regimens on CSF biochemistry and seizure control in epileptic patients. Br J Clin Pharmacol. 1989; 27(suppl):79-85.

2. Riekkinen PJ, Ylinen A, Halonen T, Sivenius J, Pitkanen A. Cerebrospinal fluid, GABA and seizure control with vigabatrin. BrJ Clin Pharmacol. 1989;27(suppl):87-94.

3. Kälviäinen R, Aikiä M, Saukkonene AM, Mervaala E, Riekkinen PJ. Vigabatrin versus carbamazepine monotherapy in newly diagnosed patients with epilepsy. Arch Neurol. 1995;52:989-996.

4. Hancock E, Osborne J, Milner P. Treatment of infantile spasms. Cochrane Database Systematic Reviews 2003;CD0011770.

5. Mackay MT, Weiss SK, Adams-Webber T, et al. Practice parameter: medical treatment of infantile spasms: report of the American
Academy of Neurology: Child Neurology Society. Neurology. 2004;62:1668-1681.

6. Elterman RD, Shields WD, Mansfield KA, Nakagawa J. Randomized trial of vigabatrin in patients with infantile spasms. Neurology. 2002;57:1416-1421.

7. Schechter PJ. Clinical pharmacology of vigabatrin. Br J Clin Pharmacol. 1989;27(suppl):19-22.

8. Eke T, Talbot JF, Lawden MC. Severe persistent visual field constriction associated with vigabatrin. BMJ. 1997;314:180-181.

9. Krauss GL, Johnson MA, Miller NR. Vigabatrin-associated retinal cone system dysfunction: electroretinogram and ophthalmic findings. Neurology. 1998;50:614-618.

10. Arndt CF, Derambure P, Defoort-Dhellemmes S, Hache JC. Outer retinal dysfunction in patients treated with vigabatrin. Neurology. 1999;52:1201-1205.

11. Daneshvar H, Racette L, Coupland SG, Kertes PJ, Guberman A, Zackon D. Symptomatic and asymptomatic visual loss in patients taking vigabatrin. Ophthalmology. 1999;106:1792-1798.

12. Kalviainen R, Nousiainen I, Mantyjarvi M, Nikoskelainen E, Partanen K, Riekkinen P. Vigabatrin, a GABAergic antiepileptic drug, causes concentric visual field defects. Neurology. 1999;53:922926.

13. Miller NR, Johnson MA, Paul SR, Girkin CA, Perry JD, Endres M. Visual dysfunction in patients receiving vigabatrin. Neurology. 1999;53:2082-2087.

14. Lawden MC, Eke T, Degg C, Harding GFA, Wild JM. Visual field defects associated with vigabatrin therapy. J Neurol Neurosurg Psychiatry. 1999;67:716-722.

15. Wild JM, Martinez C, Reinshagen G, Harding GFA. Characteristics of a unique visual field defect attributed to vigabatrin. Epilepsia. 1999;40:1784-1794.

16. Stefan H, Bernatik J, Knorr HLJ. Visual field constriction and antiepileptic drug treatment. Neurol Psychiatry Brain Res. 2000;7: 185-190.

17. Fledelius HC. Vigabatrin-associated visual field constriction in a longitudinal series: reversibility suggested after drug withdrawal. Acta Ophthalmol Scand. 2003;81:41-45.

18. Nicolson A, Leach JP, Chadwick DW, Smith DF. The legacy of vigabatrin in a regional epilepsy clinic. J Neurol Neurosurg Psychiatry. 2002;73:327-329.

19. Schmitz B. Visual field defects and vigabatrin: an update. Aktuelle Neurol. 2002;29:S24-S27.

20. Harding GFA, Wild JM, Robertson KA, Rietbrock S, Martinez C. Separating the retinal electrophysiologic effects of vigabatrin: treatment versus field loss. Neurology. 2000;55:347-352.

21. Newman WD, Tocher K, Acheson JF. Vigabatrin associated visual field loss: a clinical audit to study prevalence, drug history and effects of drug withdrawal. Eye. 2002;16:567-571.

22. Frisen L, Malmgren $\mathrm{K}$. Characterization of vigabatrin-associated optic atrophy. Acta Ophthalmol Scand. 2003;81:466-473.

23. Bunsic JR, Westall CA, Panton CM, Munn JR, MacKeen LD, Logan WJ. Characteristic retinal atrophy with secondary 'inverse' optic atrophy identifies vigabatrin toxicity in children. Ophthalmology. 2004;111:1935-1942.

24. Ravindran J, Blumbergs P, Crompton J, Pietris G, Waddy H. Visual field loss associated with vigabatrin: pathological correlations. J Neurol Neurosurg Psychiatry. 2001;70:787-789.

25. Choi HJ, Kim DM. Visual field constriction associated with vigabatrin: retinal nerve fibre photographic correlation. $J$ Neurol Neurosurg Psychiatry. 2004;75:1395.

26. Viestenz A, Viestenz A, Mardin CY. Vigabatrin-associated bilateral optic nerve atrophy with visual field construction: a case report and a survey of the literature. Ophthalmologue. 2003;100:402405.

27. Paunescu LA, Schuman JS, Price LL, et al. Reproducibility of nerve fibre layer thickness, macular thickness and optic nerve head measurements using StratusOCT. Invest Ophthalmol Vis Sci. 2004; 45:1716-1724.

28. Hardus P, Verduin WM, Engelsman M, et al. Visual field loss associated with vigabatrin: quantification and relation to dosage. Epilepsia. 2001;42:262-267. 
29. Harding GFA, Robertson KA, Edson AS, Barnes P, Wild JM. Visual electrophysiological effect of a GABA transaminase blocker. Doc Ophthalmol. 1999;97:179-188.

30. Comaish IF, Gorman C, Brimlow GM, Barber C, Orr GM, Galloway $\mathrm{N}$. The effects of vigabatrin on electrophysiology and visual field defects in epileptics: a controlled study with a discussion of possible mechanisms. Doc Opbthalmol. 2002;104:195-212.

31. Besch DA, Kurtenbach E, Apfelstedt-Sylla B, et al. Visual field constriction and electrophysiological changes associated with vigabatrin. Doc Opbthalmol. 2002;104:151-170.

32. McDonagh J, Stephen LJ, Dolan FM, et al. Peripheral retinal dysfunction in patients taking vigabatrin. Neurology. 2003;61:16901694.

33. Nousiainen I, Kalviainen R, Mantyjarvi M. Contrast and glare sensitivity in epilepsy patients treated with vigabatrin or carbamazepine monotherapy compared with healthy volunteers. $\mathrm{Br} \mathrm{J} \mathrm{Oph-}$ thalmol. 2000;84:622-625.

34. Roff Hilton EJ, Cubbidge RP, Hosking SL, Betts TA, Comish IF Patients treated with vigabatrin exhibit central visual function loss. Epilepsia. 2002;43:1351-1359.

35. Nousiainen I, Kalviainen R, Mantyjarvi M. Color vision in epilepsy patients treated with vigabatrin or carbamazepine monotherapy. Ophthalmology. 2000;107:884-888.
36. Ozkul Y, Gurier B, Uckardes A, Bozlar S. Visual functions in epilepsy patients on valproate monotherapy. J Clin Neurosci. 2002;9:247-250.

37. Arndt CF, Salle M, Derambure PH, Defoort-Dhellemmes S, Hache JC. The effect on vision of associated treatments in patients taking vigabatrin: carbamazepine versus valproate. Epilepsia. 2002; 43 : 812-817.

38. Sommer A, Katz J, Quigley HA, et al. Clinically detectable nerve fibre atrophy precedes the onset of glaucomatous field loss. Arch Opbthalmol. 1991;109:77-83.

39. Garway-Heath DF, Poinoosaumy D, Fitzke FW, Hitchings RA. Mapping the visual field to the optic disc in normal tension glaucoma eyes. Opbthalmology. 2000;107:1809-1815.

40. Schuman JS, Pedut-Kloizman T, Hertzmark E, et al. Reproducibility of nerve fibre layer thickness measurements using optical coherence tomography. Ophthalmology. 1996;103:1889-1898.

41. Carpiento P, Ciancaglini M, Zuppardi E, Falconio G, Doronzo E, Mastropasqua L. Reliability of nerve fibre layer thickness measurements using optical coherence tomography in normal and glaucomatous eyes. Ophthalmology. 2003;110:190-195.

42. Cense B, Chen TC, Park BH, Pierce MC, de Boer JF. Thickness and birefringence of healthy retinal nerve fibre layer tissue measured with polarization-sensitive optical coherence tomography. Invest Ophthalmol Vis Sci. 2004;45:2606-2612. 